\title{
Control inhibitorio y monitorización en población infantil con TDAH
}

\section{Inhibitory Control and monitoring in Child Population with ADHD Controle inibitório e monitorização em população infantil com TDAH}

\author{
Carlos Ramos-Galarza*, Claudia Pérez-Salas** \\ " Universidad Internacional SEK del Ecuador, Quito, Ecuador. \\ ${ }^{* *}$ Universidad de Concepción, Concepción, Chile.
}

Doi: http://dx.doi.org/10.12804/revistas.urosario.edu.co/apl/a.4195

\section{Resumen}

El trastorno por déficit de atención con hiperactividad (TDAH) ha sido asociado a déficits de las funciones ejecutivas, sin embargo, aún no existe claridad respecto a cuál es el perfil cognitivo en el subtipo combinado (TDAH-C). Los objetivos de este estudio fueron comparar el control inhibitorio y la monitorización de estudiantes con y sin TDAH-C, y analizar la correlación entre tareas experimentales y escalas comportamentales de evaluación de las funciones ejecutivas mencionadas. El estudio tuvo 68 participantes, quienes conformaron un grupo experimental de 34 estudiantes con TDAH-C y un grupo control de 34 estudiantes con desarrollo típico. Los instrumentos de medición fueron las escalas comportamentales BRIEF, BIS 11-c y ADHD Rating Scale IV, y los experimentos Go/No-Go, Simon y Stroop Victoria. Se encontró que el grupo experimental con TDAH presenta déficits en las funciones ejecutivas evaluadas, y que las escalas comportamentales y los experimentos evalúan diferentes niveles del funcionamiento ejecutivo.
Palabras clave: control inhibitorio; funciones ejecutivas; monitorización; neuropsicología; trastorno por déficit de atención con hiperactividad.

\section{flbstract}

\begin{abstract}
Attention deficit hyperactivity disorder (ADHD) has been associated with deficits in executive functions, but no cognitive profile is clearly associated with the combined subtype (ADHD-C). The goals of this study were to compare the inhibitory control and the monitoring of students with and without ADHD-C, and to analyze the correlation between experimental tasks and scales for behavioral evaluation with the referred executive functions. The study had 68 participants, including an experimental group of 34 students with ADHD-C and a control group of 34 students reflecting typical development. The instruments for measurement were the behavioral scales BRIEF, BIS 11-c, and ADHD Rating Scale IV, and the experiments were based on Go/No-Go, Simon, and Victoria Stroop tasks. It was found that the
\end{abstract}

* Carlos Ramos-Galarza, Departamento de Psicología Clínica, Universidad Internacional SEK del Ecuador, Quito, Ecuador; ${ }^{* *}$ Claudia Pérez-Salas, Departamento de Psicología, Universidad de Concepción, Concepción, Chile.

La correspondencia relacionada con este artículo debe ser enviada a Carlos Ramos-Galarza, Calle Alberto Einstein s/n y 5 ta. transversal. Teléfono: 593 3974800. Correo electrónico: carlos.ramos@uisek.edu.ec

Cómo citar este artículo: Ramos-Galarza, C. \& Pérez-Salas, C. (2017). Control inhibitorio y monitorización en población infantil con TDAH. Avances en Psicología Latinoamericana, 35(1), 117-130. doi: http://dx.doi.org/10.12804/revistas.urosario. edu.co/apl/a.4195 
experimental group with ADHD presented deficits in the executive functions evaluated, and that the behavioral scales and the experiments evaluated different levels of executive function.

Keywords: Inhibitory control; executive functions; monitoring; neuropsychology; attention deficit hyperactivity disorder.

\section{Resumo}

O transtorno por déficit de atenção com hiperatividade (TDAH) tem sido associado a déficits das funções executivas, no entanto, ainda não existe claridade em relação a qual é o perfil cognitivo no subtipo combinado (TDAH-C). Os objetivos deste estudo foram comparar o controle inibitório e monitorização de estudantes com e sem TDAH-C, e analisar a correlação entre tarefas experimentais e escalas comportamentais de avaliação das funções executivas mencionadas. $\mathrm{O}$ estudo conformou-se por 68 participantes, quem conformaram um grupo experimental de 34 estudantes com TDAH-C e um grupo controle de 34 estudantes com desenvolvimento típico. Os instrumentos de medição foram as escalas comportamentais BRIEF, BIS 11-c e ADHD Rating Scale IV, e os experimentos Go/No-Go, Simon e Stroop Victoria. Encontrou-se que o grupo experimental com TDAH apresenta déficits nas funções executivas avaliadas e que as escalas comportamentais e experimentos avaliam diferentes níveis do funcionamento executivo. Palavras-chave: controle inibitório; funções executivas; monitorização; neuropsicologia; transtorno por déficit de atenção com hiperatividade.

\section{Introducción}

El trastorno por déficit de atención con hiperactividad (TDAH) es definido por la Asociación Americana de Psiquiatría (APA) como un trastorno del neurodesarrollo que presenta un cuadro clínico caracterizado por un patrón elevado de impulsividad, excesiva actividad motora y déficit en el proceso atencional, signos y síntomas que generan un deterioro clínicamente significativo en el niño que lo presenta (APA, 2013).

La sintomatología del TDAH se configura en dos dimensiones: el déficit de atención y la impulsividad/hiperactividad (APA, 2013). Según Barkley (2011), en la primera dimensión se presentan alteraciones en el mantenimiento de la atención para realizar actividades académicas o lúdicas; la segunda dimensión se refiere a la abundancia de movimientos sin un fin determinado y un comportamiento irreflexivo.

Según la actual clasificación del TDAH en el DSM-5 (APA, 2014), se indica que éste se descompone en tres subtipos: a) TDAH-C, que engloba sintomatología de déficit atencional e impulsividad/hiperactividad; b) TDAH predominante inatento, que se conforma por sintomatología de desatención; y c) TDAH predominante hiperactivo/impulsivo, que se compone por sintomatología impulsiva e hiperactiva.

En cuanto a la prevalencia de los subtipos del TDAH, se ha reportado que la sintomatología relacionada al subtipo combinado es la que se presenta con mayor frecuencia en el medio educativo y clínico, siendo aproximadamente prevalente entre el $3 \%$ y 10\% de la población (APA, 2013; De la Barra, Vicente, Saldivia, \& Melipillan, 2013).

Diversos estudios (Halperin, Trampush, Miller, Marks, \& Newcorn, 2008; Vaughn et al., 2011; Vélez Van Meerbeke, Zamora, Guamzán, López, \& Talero-Gutiérrez, 2013; Winther, Egeland, Norman, Tore, \& Øie, 2014) han reportado que los niños que presentan TDAH-C evidencian más déficits a nivel cognitivo y comportamental, constituyéndose en el subtipo de mayor frecuencia e impacto en el medio educativo, familiar y social.

La etiología del TDAH ha sido descrita como multicausal, ya que no se ha llegado a un consenso científico que permita explicar con contundencia los factores causales de este complejo cuadro clínico (Servera, 2005).

Desde la neuropsicología se ha propuesto que la sintomatología del TDAH sería una consecuencia 
de un déficit en las funciones ejecutivas, lo cual ha sido reportado en diferentes estudios y propuestas explicativas del TDAH (Barkley, 1997; Brown, 2008; Castellanos, Sonuga-Barke, \& Tannock, 2006; Salum et al., 2014).

Uno de los autores que mayor avance ha realizado en la comprensión teórica del TDAH es Barkley (1997, 2011), quien afirma que este trastorno sería producto de una afectación del control inhibitorio. Según su modelo, el mecanismo del control inhibitorio se conforma por tres factores: a) la capacidad de inhibir respuestas prepotentes o automáticas ante un evento; b) la interrupción de respuestas prepotentes o automáticas en marcha, que es el control motor que se puede presentar en las respuestas que se están ejecutando; y c) el control de la interferencia, que es la capacidad de controlar las interrupciones e interferencias de estímulos tanto ambientales como internos (Sánchez-Carpintero \& Narbona, 2004).

En este modelo se toman en consideración cuatro funciones ejecutivas que presentan una relación bidireccional en la autorregulación del comportamiento (Servera, 2005). La primera función ejecutiva es la memoria de trabajo no verbal, que posibilita la retención de la información para su utilización en respuestas contingentes ante un evento, y permite la interiorización de las actividades sensoriomotoras (Barkley, 1997). Al estar afectada en el TDAH, se presentarían déficits en la percepción retrospectiva, la capacidad de previsión, la conciencia y el dominio del tiempo, y la capacidad de imitación de un nuevo comportamiento (Hughes, Wilson, \& Emslie, 2013).

La segunda función ejecutiva comprometida es la autorregulación de la motivación, las emociones y el arousal, que engloba la capacidad para tener una representación mental coherente entre la relación del comportamiento con estados emocionales y afectivos (Barkley, 1997). Los déficits en esta función ejecutiva se reflejarían tanto en la impulsividad y la tendencia a manifestar poca regulación en la expresión emocional y motivacio- nal, como en fluctuaciones en el estado de alerta (Hughes et al., 2013). La tercera función ejecutiva es la reconstitución comportamental, conformada por la fragmentación de conductas observadas y la recombinación de sus partes para el diseño de nuevas acciones (Barkley, 1997).

Un factor que influye en el desempeño de la reconstitución comportamental es el déficit de la flexibilidad cognitiva presente en el TDAH, el cual genera dificultades en el análisis y síntesis de conductas aprendidas, y la generación de nuevas conductas adaptativas en el contexto. Esto limita la capacidad para resolver problemas mediante la combinación y creación de conductas basadas en su repertorio comportamental (Hughes et al., 2013).

La cuarta función ejecutiva es el habla autodirigida asociada a la monitorización del comportamiento, que engloba el aprender, interiorizar y seguir reglas e instrucciones, la resolución de un problema, y construir metareglas (Barkley, 1997). Su compromiso lleva a mayor dificultad para regular y monitorear el comportamiento, y este déficit es el causante de la escasa capacidad del niño con TDAH para autopreguntarse sobre la eficacia de sus acciones mientras ejecuta y termina una tarea (Hughes et al., 2013).

Según Barkley (1990), esta capacidad de tener conciencia de los objetivos y consecuencias del comportamiento dependen directamente de la capacidad lingüística, la cual, a su vez, influye en el monitoreo del propio comportamiento. Según Arango, Puerta y Pineda (2008), la monitorización es una función cognitiva de orden superior que permite al ser humano controlar y supervisar su propio rendimiento durante la realización de una tarea o inmediatamente al finalizarla, con el fin de cerciorarse que el objetivo planteado haya sido alcanzado apropiadamente.

Según varios autores (Mateo \& Villaplana, 2007; Miyake et al., 2000), la monitorización actúa en paralelo a la realización de alguna actividad, permite vigilar el adecuado y eficaz rendimiento de sus procesos cognitivos y comportamentales en 
curso, tener conciencia de posibles desviaciones de la conducta sobre una meta propuesta y permite una posible corrección y actualización de la información sobre un error o desvío del objetivo antes de ver el resultado final.

Gioia, Isquith, Guy y Kenworthy (2000) proponen que la monitorización se subdivide en varios componentes. En primer lugar, se puede analizar la monitorización como una función ejecutiva general; en segundo término, se la podría entender como un subcomponente ejecutivo de los factores regulación comportamental o metacognición, en el primero como monitorización del comportamiento social y en el segundo como monitorización en la resolución de tareas.

En relación con lo afirmado, varios autores (Ramalho, García-Señorán, \& González, 2011; Skogli, Egeland, Norman, Tore, \& Øie, 2014) han reportado que los déficits del control inhibitorio y monitorización serían las principales alteraciones cognitivas de los niños con TDAH.

Estos reportes tienen relación con lo reportado por Noordermeer, Luman y Oosterlaan (2016), quienes describen que el comportamiento autorregulado en un infante depende de zonas cerebrales frontales, relacionadas con la programación, ejecución, monitorización y verificación de su actividad, situación que no sucedería con los niños con TDAH, puesto que su comportamiento se caracteriza por dejar las actividades sin terminarlas o sin cumplir con los objetivos propuestos (APA, 2014).

En la evaluación del control inhibitorio y la monitorización se han utilizado diversas metodologías, que van desde el uso de técnicas de neuroimagen (Castellanos \& Tannock, 2002; Winstanley, Theobald, Dalley, Cardinal, \& Robbins, 2006), tareas experimentales (Bezdjian, Baker, Lozano, \& Raine, 2009), hasta cuestionarios de evaluación comportamental (Damasio, 1994; Gioia et al., 2000).

Dentro del uso de escalas de evaluación comportamental y tareas experimentales de evaluación de las funciones ejecutivas, Barkley y Fischer (2011) afirman que estas últimas serían ajenas a las escalas comportamentales, argumentando que esta disparidad entre esas dos metodologías se debe a que probablemente estén evaluando diferentes niveles de un sistema ejecutivo jerárquicamente organizado.

En tal contexto, nos proponemos dos objetivos a lograr con la presente investigación: a) comparar el control inhibitorio y la monitorización en niños con y $\sin$ TDAH-C, y b) analizar la correlación existente entre pruebas experimentales de evaluación del control inhibitorio y monitorización en relación con las escalas de valoración comportamental de esta función ejecutiva.

\section{Método}

\section{Participantes}

Tanto el grupo control como el grupo experimental estuvieron conformados por 34 participantes, con una media de edad de 9,88 años ( $D E$ $=0,50)$ para el grupo con TDAH y una media de 9,88 años $(D E=0,34)$ para el grupo sin TDAH. En la organización del grupo control se consideró la selección de niños y niñas con las mismas características de los experimentales, salvo la presencia del TDAH-C. Según el análisis de comparación de medias de la variable edad, se encuentra que ésta no es diferente en cada uno de los grupos $(t=0,00$, $p=1,00)$. En cuanto al sexo, el grupo con TDAH estuvo compuesto por 23 hombres $(67,65 \%)$ y 11 mujeres (32,35\%). El grupo sin TDAH se conformó con 17 hombres $(50 \%)$ y 17 mujeres $(50 \%)$. Esta variable no presenta diferencias en relación con ambos grupos $\left(x^{2}[1, \mathrm{~N}=68]=2,186, p=0,139\right)$. Los participantes pertenecen al sistema educativo público de Ecuador. El nivel socioeconómico es medio y medio bajo. Para el ingreso de los participantes en determinado grupo se determinaron criterios de inclusión y exclusión.

En el grupo experimental se consideraron los criterios de inclusión y exclusión: a) poseer el diagnóstico de TDAH-C emitido por el Departamento 
de Psicología de la institución educativa a la que pertenecían; b) diagnóstico de TDAH-C ratificado por los equipos de atención psicopedagógica estatales; c) presentar evaluación de la inteligencia dentro de parámetros normales; d) no presentar algún tipo de discapacidad sensorial o motriz; e) no presentar antecedentes de graves trastornos afectivos; f) no recibir tratamiento farmacológico; y g) contar con consentimiento y asentimiento informado para participar en la investigación.

Los criterios de inclusión y exclusión considerados para el grupo control fueron: a) no poseer diagnóstico de TDAH-C o antecedentes de algún tipo de cuadro psicopatológico; b) no presentar antecedentes de discapacidad intelectual o presencia de algún tipo de déficit cognitivo; c) no poseer algún tipo de discapacidad sensorial o motriz; d) no recibir tratamiento farmacológico por algún trastorno psiquiátrico; y e) contar con consentimiento y asentimiento informado para participar en la investigación.

Los participantes del grupo experimental y control fueron contactados en tres instituciones del sistema educativo público de Ecuador. En todos los casos se coordinó con el Departamento de Psicología de las instituciones, quienes aportaron en la detección de los participantes de ambos grupos. A pesar de contar con el diagnóstico del TDAH-C, se aplicaron dos escalas (ADHD Rating Scale IV y BIS 11-c) para confirmar dicho diagnóstico en el grupo experimental y descartarlo en el grupo control.

\section{Instrumentos}

\section{Tareas experimentales}

Tarea experimental Go/No-Go es una actividad de inhibición de la respuesta en la que ésta debe ser ejecutada o inhibida (Bezdjian et al., 2009). Durante esta tarea los participantes debieron responder a un estímulo Go (P en la primera fase y $\mathrm{R}$ en la segunda fase) e inhibir la respuesta a un estímulo
No-Go ( $\mathrm{R}$ en la primera fase y $\mathrm{P}$ en la segunda fase) (Mueller \& Piper, 2014). La tarea contiene 362 ensayos, con un tiempo total de duración aproximado de 9 minutos. Los estímulos (R-P) se presentan en una matriz 2 X 2 que contiene cuatro estrellas donde se presentan las letras mencionadas (figura 1). De este experimento se cuantificaron dos variables: a) número de errores ante el estímulo de no respuesta (No-Go) y b) tiempo de reacción.

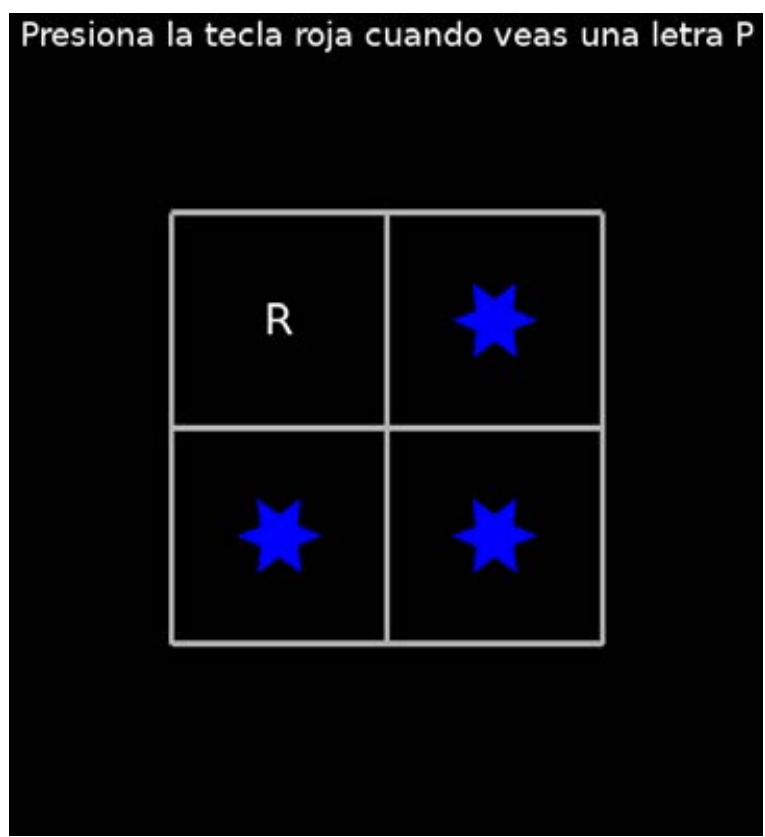

Figura 1. Captura de pantalla de la primera fase del experimento Go/No-Go, en el que el estímulo Go es la letra $\mathrm{P}$

Fuente: Mueller \& Piper, 2014

La tarea experimental del efecto Simon (Mueller \& Piper, 2014) es una tarea cognitiva de inhibición de una tendencia de la respuesta (Kunde \& Stocker, 2002). En ésta se presentan en la pantalla un círculo de color rojo o azul, uno a la vez. En el centro de la pantalla existe una cruz dividiéndola en un hemicampo derecho y un hemicampo izquierdo (figura 2). El número de ensayos es 140, el tiempo de realización del experimento es entre 4 y 6 minutos. Según Barkley (2003), en esta tarea los seres humanos tenemos una tendencia a responder 
al hemicampo en el cual se encuentre el estímulo. Por tanto, se definió la respuesta de cada participante en relación con el color del círculo presentado, de manera que cuando se presentó un círculo de color azul el participante debía responder (con un joystick programado para el experimento) hacia la derecha, y hacia la izquierda cuando se presentaba el círculo de color rojo, sin importar el hemicampo en el cual se presentara. De esta tarea se cuantificaron dos variables: a) total de errores Simon y b) tiempo de reacción global.

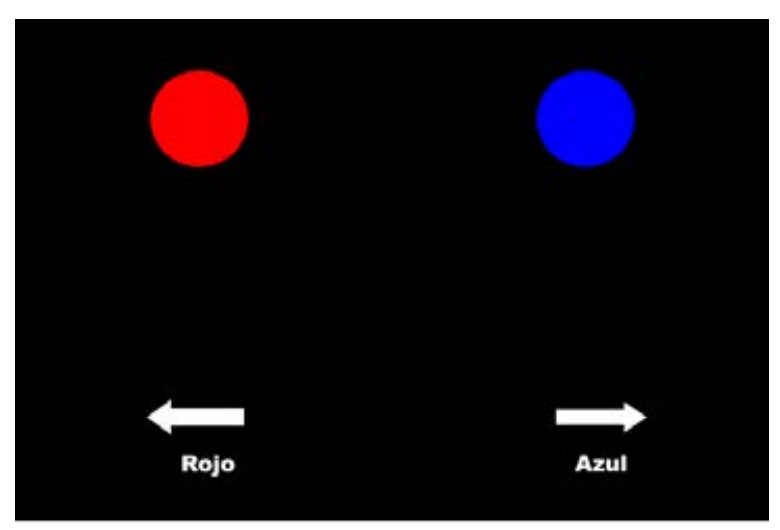

Figura 2. Captura de pantalla del experimento Simon, en el que se presentan los estímulos y posibles respuestas

Fuente: Mueller \& Piper, 2014

La tarea experimental Stroop Victoria (Mueller \& Piper, 2014) es una tarea de control inhibitorio basada en el paradigma Stroop clásico en una versión breve (Troyer, Leach, \& Strauss, 2006). Ésta consta de tres fases (cada una de 26 estímulos). En la primera, el participante debe presionar los botones adecuados que indiquen el color (azul, rojo, amarillo y verde) de los círculos presentados en el monitor (figura 3); en la segunda fase debe presionar el botón correspondiente al color de la tinta con la cual se encontraba escrita la palabra (una diferente al nombre del color); y en la fase final se presenta la interferencia, en la cual se encuentra el nombre de un color y el sujeto debe seleccionar el botón que indica el color de la tinta de la palabra, para lo cual debe inhibir la tendencia natural de lectura (Malek, Hekmati, Amiri, Pirzadeh, \& Gholizadeh, 2013). En este experimento se obtuvieron dos variables: a) total de movimientos Stroop y b) errores de intrusión Stroop.

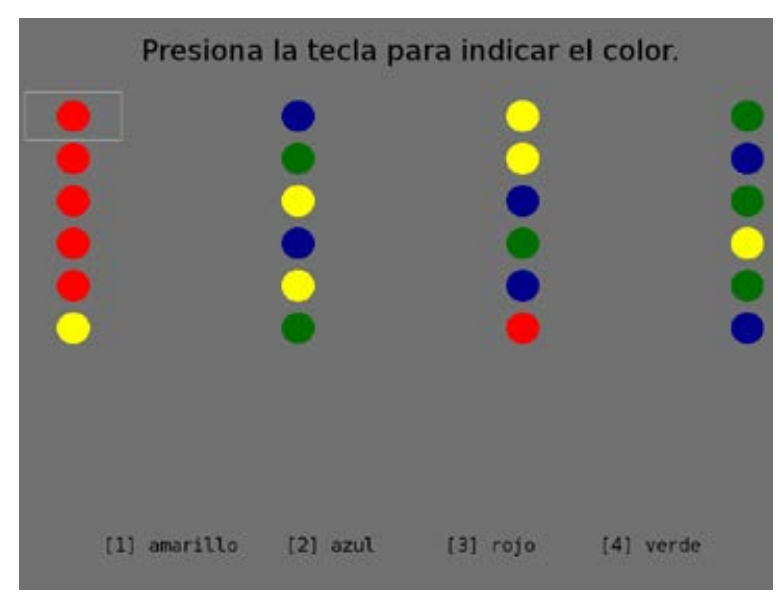

Figura 3. Captura de pantalla de la primera fase del experimento Stroop Victoria en el que se presentan los estímulos de colores y las opciones de respuestas

Fuente: Mueller \& Piper, 2014

\section{Escalas comportamentales}

Se usó el cuestionario de evaluación de las funciones ejecutivas BRIEF versión para profesores (Gioia et al., 2000), el cual consta de dos índices: metacognición y regulación comportamental. Está compuesto por 8 escalas y 86 ítems, los cuales se califican con tres opciones de repuesta: nunca (un punto), a veces (dos puntos) y con frecuencia (tres puntos). En el estudio se utilizaron las subescalas de control inhibitorio y monitorización. Cada una de las escalas consta de 10 ítems. En estudios previos se ha reportado que la consistencia interna de inhibición es $\alpha=0,85$ y monitorización $\alpha=0,60$ (García, González-Pienda, Rodríguez, Álvarez, \& Álvarez, 2014) o $\alpha=0,94$ y $\alpha=0,85$, en el orden anterior (Gioia et al., 2000). En la presente investigación, el coeficiente de alfa de Cronbach de la escala de monitorización fue de $\alpha=0,87$ y en la escala de inhibición $\alpha=0,86$. Con este cuestionario se valoraron las variables: a) control inhibitorio; 
b) monitorización general; c) monitorización del comportamiento social; y d) monitorización en la resolución de tareas.

Además, se utilizó el ADHD Rating Scale IV versión para profesores (Du Paul et al., 1997). Es un cuestionario basado en los criterios diagnósticos del DSM-5 (APA, 2013) para el TDAH. Se usó la versión para profesores. Consta de 18 ítems que valoran las variables: a) déficit de atención, b) impulsividad/hiperactividad y c) total TDAH. Cada ítem se lo valora con cero cuando la conducta tiene una frecuencia de nunca, con uno cuando es a veces, dos cuando es frecuentemente y tres para muy frecuentemente. En una investigación previa se ha reportado una consistencia interna de la escala de déficit de atención $\alpha=0,95$ y en la escala de hiperactividad/impulsividad $\alpha=0,94$ (Servera \& Cardo, 2007). En la presente investigación el parámetro de consistencia interna para la escala déficit de atención fue $\alpha=0,95$ y en la escala de hiperactividad/impulsividad fue $\alpha=0,96$.

Por último, se empleó la Escala de Impulsividad de Barrat BIS 11-c, la cual es un cuestionario de autorreporte que permite evaluar las variables: (a) impulsividad cognitiva, (b) impulsividad no planificada e (c) impulsividad motora (Stanford et al., 2009). Este instrumento consta de 26 ítems que son evaluados con cero puntos cuando la conducta tiene una frecuencia de nunca, con un punto cuando es a Veces, con dos puntos cuando es regularmente y tres puntos cuando la conducta se presenta siempre. En investigación previa sobre este instrumento se ha reportado parámetros de consistencia interna para impulsividad cognitiva $\alpha=0,59$, impulsividad motora $\alpha=0,74$ e impulsividad no planificada $\alpha=$ 0,72 (Chahin, Cosi, Lorenzo-Seva, \& Vigil-Colet, 2010). En el presente estudio el coeficiente de consistencia interna de la escala impulsividad motora fue $\alpha=0,73$, impulsividad cognitiva $\alpha=0,65 \mathrm{y}$ escala impulsividad no planificada fue $\alpha=0,66$.

\section{Procedimiento}

Los experimentos se ejecutaron en dos computadoras portátiles con igual procesador, monitor y caja de botones. En primer lugar, se procedió a realizar la programación de los experimentos (traducción de instrucciones de inglés a español, adición de imágenes, número de estímulos y colores); esta tarea fue realizada por el autor principal del estudio. Después, se evaluaron los experimentos mediante el juicio experto de tres profesionales con título de posgrado en Neuropsicología y amplia experiencia en la evaluación de las funciones ejecutivas. Una vez que se contó con los experimentos en las condiciones idóneas para ser utilizados, se procedió a realizar las evaluaciones. El contexto en el cual se aplicaron los experimentos y escalas de evaluación comportamental se enmarca dentro de los parámetros éticos de Helsinki para la investigación con seres humanos (Williams, 2008) y en todos los casos se presentaron las mismas condiciones. Es importante mencionar que la presente investigación contó con la aprobación del comité de ética del Doctorado en Psicología de la Universidad de Concepción, Chile.

\section{Análisis de datos}

Todos los análisis se realizaron en el programa IBM SPSS Statistics versión 20. En primer lugar, se hizo el análisis descriptivo de las variables evaluadas mediante medidas de tendencia central y dispersión. En segunda instancia se realizó el análisis paramétrico $t$ de Student para comparar las variables medidas mediante las escalas y los experimentos del estudio. Finalmente, para cumplir con el objetivo de verificar asociaciones en las pruebas neuropsicológicas experimentales con las escalas comportamentales se utilizó el proceso de correlación de Pearson. 


\section{Resultados}

En primer lugar, se procedió a aplicar los instrumentos ADHD Rating Scale y la Escala BIS 11-c con la finalidad de confirmar el diagnóstico de TDAH en el grupo experimental con TDAH y el no cumplimiento del cuadro en los participantes del grupo de control de estudiantes sin TDAH. Cabe resaltar que los participantes con TDAH poseen un diagnóstico clínico y psicométrico previo.

El primer aspecto que se analizó de los datos fue el cumplimiento de los criterios de normalidad, en el que se encontró que los niveles de asimetría y curtosis, gráficos de normalidad y demás análisis estaban con características de normalidad y homocedasticidad adecuada para realizar el análisis estadístico. Los resultados encontrados en la escala ADHD indican la presencia de diferencias significativas en las variables: a) total de síntomas de TDAH $t(66,2)=11,50, p<0,001$, donde el grupo con TDAH presentó un mayor puntaje $(M=36,08$, $D E=7,67)$ en relación con el grupo sin TDAH $(M$ $=12,74, D E=6,70)$; b) déficit de atención $t(66,2)$ $=9,80, p<0,001$, siendo el grupo con TDAH el que presentó mayores niveles en la cuantificación de la variable $(M=18,67, D E=6,63)$, en relación con el grupo sin TDAH $(M=4,85, D E=2,10)$; y c) impulsividad/hiperactividad $t(66,2)=10,14, p<$ 0,001 , donde el grupo con TDAH presentó mayor puntuación $(M=17,41, D E=2,82)$, en relación con el grupo sin TDAH $(M=7,91, D E=2,79)$.

En cuanto a la Escala BIS 11-c se encontraron diferencias significativas en las variables: a) impulsividad no planificada $t(66,2)=-3,98, p<0,001$, siendo el grupo con TDAH el que presentó mayor deterioro $(M=14,97, D E=4,75)$, en relación con el grupo control $(M=18,85, D E=3,11)$, es importante mencionar que en esta variable (a diferencia de todas las variables del estudio) a menor puntaje mayor déficit; y en b) impulsividad motora $t(66,2)$ $=6,31, p<0,001$, donde el grupo con TDAH presentó mayor dificultad $(M=14,50, D E=5,23)$, en relación con el grupo sin TDAH $(M=5,88, D E=$ $5,00)$. En impulsividad cognitiva no se encontraron diferencias $t(66,2)=-1,53, p<0,13$.

En la tabla 1 se presentan los valores descriptivos y la comparación mediante $t$ de Student del funcionamiento del control inhibitorio y la monitorización que se valoraron mediante los experimentos y las escalas comportamentales. Mediante el procedimiento $t$ de Student se encontraron diferencias estadísticamente significativas en las siguientes medidas experimentales: errores ante el estímulo No-Go, errores de intrusión en la tarea Stroop, total de movimientos en la tarea Stroop y total de errores en la tarea Simon. No se encontraron diferencias significativas en tiempo de reacción en la tarea Go/No-Go y tiempo de reacción en la tarea Simon. En la comparación mediante las escalas comportamentales, se encontraron diferencias en las variables monitorización general, control inhibitorio, monitorización en la resolución de tareas y monitorización del comportamiento social. En la tabla 1 se presentan los resultados del $t$ test y su significancia.

Se encontraron correlaciones estadísticamente significativas entre los errores No-Go con las medidas de las escalas comportamentales: impulsividad no planeada, monitorización general, monitorización en la resolución de tareas, monitorización del comportamiento social, control inhibitorio, déficit de atención, impulsividad/hiperactividad y total TDAH. El total de movimientos en el experimento Stroop correlacionó con las medidas de las escalas impulsividad motora, monitorización general y monitorización del comportamiento social. El total de errores en el experimento Simon correlacionó con impulsividad motora, impulsividad no planificada, monitorización general, monitorización en la resolución de tareas, déficit de atención y total TDAH. El tiempo de reacción en la tarea Simon correlacionó con monitorización en la resolución de tareas y déficit de atención. El tiempo de reacción en el experimento Go/No-Go y errores de intrusión 
Tabla 1

Estadísticos descriptivos de los grupos con y $\sin T D A H$

\begin{tabular}{lcccc}
\hline & Con TDAH & Sin TDAH & & \\
\cline { 2 - 5 } & Media $(\mathrm{DE})$ & Media $(\mathrm{DE})$ & $t$ de Student & Tamaño del efecto $(d)$ \\
\hline Errores No-Go & $50,65(27,07)$ & $33,44(13,54)$ & $3,32 * *$ & 0,37 \\
\hline Tiempo de reacción Go/NoGo & $566,54(109,19)$ & $558,11(75,14)$ & 0,37 & 0,04 \\
\hline Errores de intrusión Stroop & $3,35(3,57)$ & $1,65(1,50)$ & $2,50 *$ & 0,29 \\
\hline Total de movimientos Stroop & $88,97(20,05)$ & $79,76(7,78)$ & $2,50 *$ & 0,29 \\
\hline Total de errores Simon & $20,76(14,65)$ & $12,97(8,86)$ & $2,65 *$ & 0,31 \\
\hline Tiempo de reacción Simon & $913,44(281,23)$ & $808,43(148,42)$ & 1,93 & 0,23 \\
\hline Monitorización general & $23,00(3,83)$ & $15,30(3,45)$ & $8,31 * *$ & 0,71 \\
\hline Control inhibitorio & $24,10(6,76)$ & $13,33(3,42)$ & $8,05 * *$ & 0,70 \\
\hline Monitorización en la resolución de tareas & $9,62(2,25)$ & $5,57(1,52)$ & $8,36 * *$ & 0,71 \\
\hline Monitorización del comportamiento social & $13,37(2,28)$ & $9,72(2,70)$ & $5,69 * *$ & 0,57 \\
\hline$*$ Comparación significativa $<0,05$ & & & & \\
$* *$ Comparación significativa $<0,001$ & & & & \\
\hline
\end{tabular}

Tabla 2

Correlaciones entre escalas comportamentales y tareas experimentales

\begin{tabular}{lcccccccccc}
\hline & IMP & IMP & IMP & MON & MON & MON & COTR & DEF & IMP & TOT \\
& COG & MOT & NOPL & GEN & TR & CS & INH & AT & HIP & TDAH \\
\hline Errores No-Go & $-0,19$ & 0,12 & $-0,23^{*}$ & $0,42^{* *}$ & $0,43^{* *}$ & $0,35^{* *}$ & $0,28^{*}$ & $0,38^{* *}$ & $0,26^{*}$ & $0,34^{* *}$ \\
\hline $\begin{array}{l}\text { Tiempo de reacción } \\
\text { Go/No-Go }\end{array}$ & 0,10 & 0,12 & $-0,08$ & 0,08 & $0,24^{*}$ & $-0,07$ & 0,003 & 0,06 & 0,08 & $-0,007$ \\
\hline Errores de intrusión Stroop & $-0,08$ & 0,21 & 0,06 & 0,14 & 0,70 & 0,17 & 0,11 & 0,70 & 0,03 & 0,05 \\
\hline Total de movimientos Stroop & 0,04 & $0,25^{*}$ & 0,14 & $0,26^{*}$ & 0,21 & $0,25^{*}$ & 0,19 & 0,14 & 0,10 & 0,79 \\
\hline Total de errores Simon & $-0,18$ & $0,27^{*}$ & $-0,28^{*}$ & $0,31^{* *}$ & $0,37^{* *}$ & 0,21 & 0,21 & $0,35^{* *}$ & 0,16 & $0,27^{*}$ \\
\hline Tiempo de reacción Simon & $-0,02$ & 0,003 & $-0,01$ & 0,23 & $0,28^{*}$ & 0,15 & 0,16 & $0,26^{*}$ & 0,40 & 0,16 \\
\hline
\end{tabular}

** La correlación es significativa al nivel 0,01 (bilateral).

* La correlación es significante al nivel 0,05 (bilateral).

IMP COG: impulsividad cognitiva, IMP MOT: impulsividad motora, IM NOPL: impulsividad no planificada, MON GEN: monitorización general, MON CS: monitorización del comportamiento social, MON TR: monitorización en la solución de tareas, CONTR INH: control inhibitorio, DEF AT: déficit de atención, IMP HIP: impulsividad/hiperactividad, TOT TDAH: total TDAH.

de la tarea Stroop no presentaron correlaciones estadísticamente significativas con alguna medida de las escalas comportamentales. En la tabla 2 se presentan los coeficientes de las correlaciones realizadas y su significación.

\section{Discusión}

Nuestros resultados concuerdan con investigaciones previas (Roca, Presentación-Herrero, Miranda-Casas, \& Ortiz-Sánchez, 2014; 
Vélez-Van-Meerbeke et al., 2013; Skogli et al., 2014), en las cuales se reportó que los niños y adolescentes con TDAH presentan déficits en el control inhibitorio y en la monitorización. Además, se ha descrito que el subtipo TDAH-C es el grupo que mayor gravedad presenta en las funciones ejecutivas (Holmes et al., 2010; Salum et al., 2014).

En el paradigma Go/No-Go y en el experimento Stroop Victoria se observó un mayor número de errores de control de respuesta en el grupo TDAH. Este resultado tiene relación con lo encontrado por Romero-Ayuso, Maestú, González-Marqués, Romo-Barrientos y Andrade (2006), quienes reportaron que los niños con TDAH tienden a presentar un mayor número de errores en el control conductual en experimentos de inhibición de una respuesta motora. Este resultado es coherente con el planteamiento de Barkley (1997), quien afirma que los niños con TDAH presentan déficits en relación con el control inhibitorio, control de la interferencia o control de respuestas automáticas en marcha, lo cual confirma el deterioro clínico en dicha función ejecutiva en el grupo con TDAH.

El mayor número de movimientos realizados en el Stroop Victoria se explicaría por las dificultades que presenta el grupo con TDAH en la monitorización en la resolución de tareas, es decir, necesitan cometer más errores para que la situación externa compense la supervisión en la ejecución de la tarea, a diferencia del grupo control, que presenta una regulación intrínseca. Este hallazgo es evidente en el mayor deterioro presentado por el grupo TDAH en las diversas escalas de monitorización del BRIEF, resultados que tienen relación con el reporte realizado por Mahone et al. (2002).

La mayor tasa de errores en el experimento Simon por parte del grupo con TDAH afirma el déficit en el control inhibitorio en esta población. En tal sentido, según Shallice et al. (2002) y Slachevsky y Núñez-Huasaf (2013), los participantes con TDAH presentarían dificultades en el sistema atencional supervisor, ya que en este grupo existe una tendencia a actuar sin un control deliberado de las respuestas, a diferencia del grupo sin TDAH, en el que se observó que el control de las respuestas automáticas está conservado. Es importante recalcar que en los participantes sin TDAH, una vez que su control inhibitorio determina la emisión de una respuesta conductual de tipo consciente o deliberada en un siguiente eslabón, la monitorización supervisará la adecuada ejecución en la tarea, situación que no se observa en el grupo TDAH, ya que, además de presentar las diferencias en el experimento Simon, se observa su mayor debilitamiento en todas las dimensiones de la monitorización evaluadas mediante el BRIEF.

En los tres experimentos realizados se encontraron diferencias significativas en cuanto al desempeño del grupo con TDAH en tareas de inhibición de respuestas automáticas. Estos datos se confirman en la evaluación del control inhibitorio mediante la escala comportamental BRIEF. Los resultados son coherentes con la hipótesis de la disfunción prefrontal subyacente a los déficits del control de impulsos presentes en el TDAH (Romero-Ayuso et al., 2006; Servera, 2005), lo cual generaría que el infante presente dificultades en su autocontrol ante estímulos No-Go tanto en la vida diaria como en las tareas experimentales.

Al igual que el trabajo realizado por Bioulac et al. (2012), quienes compararon el tiempo de reacción de niños con y sin TDAH mediante una tarea educativa de realidad virtual, en el presente trabajo no se encontraron diferencias significativas en el tiempo de reacción. Consideramos que este resultado se presenta porque el niño con TDAH, más allá de evidenciar una reacción más o menos rápida, posee una gran dificultad en tener consciencia de las consecuencias de sus actos (Servera, 2005); esto se puede ver reflejado en las diferencias significativas encontradas en la escala BIS 11-c en impulsividad motora e impulsividad no planificada, que permiten afirmar las dificultades que el grupo con TDAH exhibe al momento de proyectarse a las consecuencias de sus actos, tanto a nivel per- 
sonal como en quienes lo rodean (Rodríguez \& Ros, 2004).

La correlación entre las tareas experimentales y las escalas comportamentales reportadas en este artículo, al igual que en el estudio realizado por Alloway et al. (2009), se encuentran en una magnitud mediana a pequeña. Esto nos permite indicar que, si bien es cierto que nos referimos a la evaluación del mismo control inhibitorio por diferentes metodologías, éste presentaría diferentes niveles dentro de un sistema ejecutivo jerárquicamente organizado, el cual podría ser conceptualizado, como lo proponen Barkley y Fischer (2011), como un metaconstructo.

La ausencia de correlación entre los errores de intrusión en el experimento Stroop Victoria y las escalas comportamentales sería el mejor ejemplo de la organización jerárquica de las funciones ejecutivas (Barkley \& Fischer, 2011), ya que las habilidades ejecutivas que deben ponerse en juego en una tarea experimental demandarían un control inhibitorio de diferente rendimiento, en relación con el control inhibitorio que se necesita para desempeñarse en la vida diaria.

Un aspecto final a considerar en relación con los resultados del presente estudio es que los datos obtenidos permiten aportar en la hipótesis planteada dentro de la línea de investigación de procesos de evaluación y detección del TDAH, la cual afirma que la valoración de las funciones ejecutivas, como el control inhibitorio y la monitorización, son medidas confiables y válidas en la discriminación de seres humanos con y sin TDAH (Holmes et al., 2010; Salum et al., 2014; Skogli et al., 2014; Vélez-Van-Meerbeke et al., 2013).

Esta última afirmación invita al clínico a tener presente que el diagnóstico del TDAH engloba una mayor complejidad que únicamente verifica la presencia de los síntomas que engloban el cuadro, es decir, se debería realizar un proceso diagnóstico con mayor estructuración en el que se incluya el estudio del control inhibitorio o la monitorización del niño con sospecha de TDAH.
Como línea de investigación futura es importante la realización de estudios longitudinales que permitan identificar el desarrollo del control inhibitorio y la monitorización en preescolares que presenten la sintomatología del TDAH, ya que, como afirman Barkley y Fischer (2011), los déficits ejecutivos encontrados en niños con TDAH pueden permanecer como parte del cuadro hasta la etapa adulta, afectando su desempeño educativo superior o laboral, lo que invita a realizar investigación en edades tempranas para, en la medida de lo posible, reducir la severidad del trastorno en la vida de los niños que lo presentan.

Otro aspecto que se desprende como investigación futura del control inhibitorio y la monitorización de niños con TDAH es analizar si estas funciones ejecutivas podrían interactuar en un modelo explicativo de la regulación comportamental en este trastorno. En específico, nos interesa proponer un modelo en el que la monitorización pudiera moderar la relación entre el control inhibitorio y la clínica de impulsividad/hiperactividad de estudiantes con TDAH, y, luego, analizarlo mediante un experimento.

Como limitación del presente estudio podemos manifestar que el tamaño de la muestra, que si bien es cierto nos permite afirmar que este estudio presenta una potencia estadística adecuada, se presenta con limitaciones para que el estudio pueda extrapolarse a la población general con TDAH. Uno de los principales factores para el trabajo con una muestra pequeña radica en la dificultad de localización de participantes que cuenten con un diagnóstico clínico y no presenten algún tipo de necesidad educativa especial que pudiera influir en los resultados obtenidos.

\section{Agradecimientos}

El investigador principal expresa su gratitud a la Secretaría de Educación Superior, Ciencia, Tecnología e Innovación del Ecuador (SENESCYT), por el apoyo económico recibido dentro de la beca 
para cursar estudios de Ph. D. en Psicología que financió el presente proyecto.

\section{Referencias}

Alloway, T., Gathercole, S., Holmes, J., Place, M., Elliot, J., \& Hilton, K. (2009). The diagnostic utility of behavioral checklist in identifying children with ADHD and children with working memory deficits. Child Psychiatry and Human Development, 40(3), 353-366. doi: 10.1007/ s10578-009-0131-3

American Psychiatric Association (APA) (2013). Diagnostic and Statistical Manual of Mental Disorders, Fifth Edition (DSM-5). Arlington, VA: American Psychiatric Association.

American Psychiatric Association (2014). Guía de consulta de los criterios diagnósticos del DSM5. Washington, D.C.: American Psychiatric Publishing.

Arango, O., Puerta, I., \& Pineda, D. (2008). Estructura Factorial de las funciones ejecutivas desde el Dominio Conductual. Revista Diversitas Perspectivas en Psicología, 63-77.

Barkley, R. (1997). Behavioral inhibition, sustained attention, and executive functions: Constructing a unifying theory of ADHD. Psichological Bulletin, 121(1), 65-94. doi: 10.1037/00332909.121.1.65

Barkley, R. (2003). Issues in the diagnosis of attention-deficit/hyperactivity disorder in children. Brain and Development, 25(2), 77-83.

Barkley, R. (2011). Avances en el diagnóstico y la subclasificación del trastorno por déficit de atención/hiperactividad: qué puede pasar en el futuro respecto al DSM-V. Revista de Neurología, 48(2), 101-106.

Barkley, R. \& Fischer, M. (2011). Predicting impairment in major life activities and occupational functioning hyperactive children as adults: Self-reported Executive Function (EF) Deficits Versus EF Tests. Developmen- tal Neuropsychology, 36(2), 137-161. doi: 10.1080/87565641.2010.549877

Bezdjian, S., Baker, L., Lozano, D., \& Raine, A. (2009). Assessing inattention and impulsivity in children during the Go/NoGo task. British Journal of Developmental Psychology, 27(2), 365-383. doi:10.1348/026151008X314919

Bioulac, S., Lallemand, S., Rizzo, A., Philip, P., Fabrigoule, C., \& Bouvard, M. (2012). Impact of time on task on ADHD patient's perormances in a virtual classroom. European Journal of Paediatric Neurology, 16(5), 514-521. doi: 10.1016/j.ejpn.2012.01.006.

Brown, T. (2008). ADD/ADHD and impaired executive function in clinical practice. Current Psychiatry Reports, 10(5), 407-411.

Castellanos, F., Sonuga-Barke, E., Milham, M., \& Tannock, R. (2006). Characterizing cognition in ADHD: Beyond executive dysfunction. Trends in cognitive sciences, 10(3), 117-123.

Castellanos, F. \& Tannock, R. (2002). Neuroscience of attention-deficit/hyperactivity disorder: the search for endophenotypes. Nature Reviews Neuroscience, 3(8), 617-628.

Chahin, N., Cosi, S., Lorenzo-Seva, U., \& Vigil-Colet, A. (2010). Stablity of the factor structure of Barrat's Impulsivity Scales for children across cultures: A comparison of Spain and Colombia. Psicothema, 22(4), 983-989.

Damasio, A. (1994). El error de Descartes. Santiago de Chile: Editorial Andrés Bello.

De la Barra, F., Vicente, B., Saldivia, S., \& Melipillan, R. (2013). Epidemiology of ADHD in Chilean children and adolescents. ADHD Attention Deficit and Hyperactivity Disorders, 5(1), 1-8.

García, T., González-Pienda, J., Rodríguez, C., Álvarez, D., \& Álvarez, L. (2014). Psychometric characteristics of the BRIEF scale for the assessment of executive functions in Spanish clinical population. Psicothema, 26(1), 47-52.

Gioia, G., Isquith, P., Guy, S., \& Kenworthy, L. (2000). Behavior rating inventory of executive function. Lutz: Psychological Assessment Resources. 
Halperin, J., Trampush, J., Miller, C., Marks, D., \& Newcorn, J. (2008). Neuropsychological outcome in adolescents/young adults with childhood ADHD: profiles of persisters, remitters and controls. Journal of Child Psychology and Psychiatry, 49(9), 958-966.

Holmes, J., Gathercole, S., Place, M., Alloway, T., Elliott, J., \& Hilton, K. (2010). The diagnostic utility of executive function assessments in the identification of ADHD in children. Child and Adolescent Mental Health, 15, 37-43. doi: 10.1111/j.1475-3588.2009.00536.x

Hughes, A., Wilson, F. T., \& Emslie, H. (2013). Detecting executive deficits in children with ADHD or acquired brain injury using the Behavioural Assessment of Dysexecutive Sndrome (BADS). The Irish Journal of Spychology, 34(1), 13-23.

Kunde, W. \& Stocker, C. (2002). A Simon effect for stimulus-response duration. Quartely Journal of Experimental Psychology, 55, 581-592. doi: 10.1080/02724980143000433

Mahone, E., Cirino, P., Cutting, L., Cerrone, P., Hagelthrorn, K., Hiemenz, J., \& Denckla, M. (2002). Validity of the behavior rating inventory of executive function in children with ADHD and/or Tourette syndrome. Archives of Clinical Neuropsychology, 17(7), 643-662. doi: 10.1016/ S0887-6177(01)00168-8

Malek, A., Hekmati, I., Amiri, S., Pirzadeh, J., \& Gholizadeh, H. (2013). The standarization of Victoria Stroop Color-Word Test among Iranian Bilingual Adolescents. Archives of Iranian Medicine, 16(7), 380-384. doi: 013167/AIM.004

Mateo, V. \& Vilaplana, A. (2007). Estrategias de identificación del alumno inatento e impulsivo desde el contexto escolar. Quaderns Digitals, $5,13-28$.

Miyake, A., Friedman, N., Emerson, M., Witzki, A., Howerter, A., \& Wager, T. (2000). The unity and diversity of executive functions and their contributions to complex $\mathrm{x}$ 'frontal Lobe' tasks:a latent variable analysis. Cogn Psychol, 41, 49-100. doi: 10.1006/cogp.1999.0734
Mueller, S. \& Piper, B. (2014). The Psychology Experiment Building Language (PEBL) and PEBL Test Battery. Journal of Neuroscience Methods, 222, 250-259. doi: 10.1016/j.jneumeth.2013.10.024

Noordermeer, S., Luman, M., \& Oosterlaan, J. (2016). A systematic review and meta-analysis of neuroimaging in Oppositional Defiant Disorder (ODD) and Conduct Disorder (CD) taking Attention-Deficit Hyperactivity Disorder (ADHD) into account. Neuropsychology Review, 26, 44-72.

Ramalho, J., García-Señorán, M., \& González, S. (2011). Auto-instruções: estratégia de regulação atencional da THDA. Psicologia: Reflexão e Crítica, 24(1), 180-185. doi: org/10.1590/ S0102-79722011000100021

Roca, P., Presentación-Herrero, M., Miranda-Casas, A., Mulas, F., \& Ortiz-Sánchez, P. (2014). El componente P300 como correlato neurofisiológico de la memoria de trabajo conductual en adolescentes con trastorno por déficit de atención con hiperactividad. Revista de Neurología, 58(1), S51-S56.

Rodríguez, A. \& Ros, S. (2004). Los trastornos puros. En S. Ros, M. Peris, \& R. García, Imopulsividad. (1 $1^{\mathrm{a}}$ ed.) (pp. 81-99). Barcelona: Ars Médica.

Romero-Ayuso, D., Maestú, F., González-Marqués, J., Romo-Barrientos, C., \& Andrade, J. (2006). Disfunción ejecutiva en el trastorno por déficit de atención con hiperactividad en la infancia. Revista de Neurología, 42(5), 265-271.

Salum, G., Sergeant, E., Sonuga-Barke, E., Vandekerckhove, J., Gadelha, A., Pan, P., \& Prhde, L. (2014). Specify of basic information proccesing and inhibitory control in attention deficit hyperactivity disorder. Psychological Medicine, 44, 617-631. doi: 10.1017/S0033291713000639

Sánchez-Carpintero, R. \& Narbona, J. (2004). El sistema ejecutivo y las lesiones frontales en el niño. Revista de Neurología, 39(2), 188-191. 
Servera, M. (2005). Modelo de autorregulación de Barkley aplicado al Trastorno por Déficit de Atención con Hiperactividad: una revisión. Revista de Neurología, 40(6), 358-368.

Servera, M. \& Cardo, E. (2007). ADHD Rating Scale-IV en una muestra escolar española: datos normativos y consistencia interna para maestros, padres y madres. Revista de Neurología, 45(7), 393-399.

Shallice, T., Marzocchi, G., Coser, S., DelSavio, M., Meuter, R., \& Rumiati, R. (2002). Executive function profile of children with attention deficit hyperactivity disorder. Developmental Neuropsychology, 21, 43-71. doi: 10.1016/S08876177(03)00122-7

Skogli, E., Egeland, J., Norman, P., Tore, K., \& Øie, M. (2014). Few differences in hot and cold executive functions in children and adolescents with combined and inattentive subtypes of ADHD. Child Neuropsychology: A Journal on Normal and Abnormal Development in Childhood and Adolescence, 20(2), 162-181. doi: 10.1080/09297049.2012.753998

Slachevsky, A. \& Núñez-Huasaf, J. (2013). Procesos ejecutivos y adaptación del comportamiento. En J. Lavados \& A. Slachevsky, Neuropsicología de los Procesos Mentales (pp. 189-209). Santiago de Chile: Editorial Mediterráneo.

Stanford, M., Mathias, C., Dougherty, D., Lake, S., Anderson, N., \& Patton, J. (2009). Fifty years of the Barratt Impulsiveness Scale: An update and review. Personality and Individual Differences, 47, 385-395.

\section{Fecha de recepción: septiembre 16, 2015 Fecha de aprobación: abril 23, 2016}

Troyer, A., Leach, L., \& Strauss, S. (2006). Aging and response inhibition: Normative data for the Victoria Stroop Test. Aging, Neuropsychology, and Cognition, 13, 20-35. doi: 10.1016/j.neuropsychologia.2014.11.005

Vaughn, A., Epstein, J., Rausch, J., Altaye, J., Newcorn, J., Hinshaw, S., \& Wigal, T. (2011). Relation between outcomes on a continuous performance test and ADHD symptoms over time. Journal of Abnormal Child Psychology, 39, 853-864.

Vélez Van Meerbeke, A., Zamora, I., Guzmán, B., López, C., \& Talero-Gutierrez, C. (2013). Evaluación de las funciones ejecutivas en una población escolar con síntomas de déficit de atención e hiperactividad. Neurología, 28(6), 348-355. doi: 10.1016/j.nrl.2012.06.011

Williams, J. (2008). Revising the declaration of He1sinki. World medical journal, 54(4), 120-122.

Winstanley, C., Theobald, E., Dalley, J., Cardinal, R., \& Robbins, T. (2006). Double dissociation between serotonergic and dopaminergic modulation of medial prefrontal and orbitofrontal cortex during a test of impulsive choice. Cereb Cortex, 16(1), 106-114. doi: 10.1093/cercor/ bhi088

Winther, E., Egeland, J., Norman, P., Tore, K., \& Øie, M. (2014). Few differences in hot and cold executive functions in children and adolescents with combined and inattentive subtypes of ADHD. Child Neuropsychology: A Journal on Normal and Abnormal Development in Childhood and Adolescence, 20(2), 162-181. 